\title{
Smart devices and services for Smart City
}

\author{
Giuseppe Di Leo, Matteo Ferro, Consolatina Liguori, \\ Antonio Pietrosanto \\ Dep. of Industrial Engineering, Università di Salerno, Italy \\ tliguori@unisa.it
}

\author{
Vincenzo Paciello \\ Dep. of Electronic and Information Engineering, Universita \\ di Cassino e del Lazio Meridionale, Italy \\ v.paciello@unicas.it
}

\begin{abstract}
Citizen quality of life can be improved through facilities and services that have been thought to ease citizen interaction with municipal authorities, offices and structures. All technologies and devices, used for developing these facilities, are the pillars of the Smart City idea: a City that adapts itself, at least in part, to citizens' needs. Advanced Metering Infrastructure (AMI) could become the backbone of all the smart city projects. Other public services can be loaded on AMI's to be smart and thus helping to find the affordability of investments. The paper deals with this topic by describing devices and results of a pilot project, which has been carried out in an Italian middle city (Salerno), to experience the use of RF $169 \mathrm{MHz}$ wM-bus based AMI. Experimental results regarding a set of about 2500 installed devices for gas and water metering, car parking management and elder teleassistance, will be reported in detail to show convenience and problems of this approach.
\end{abstract}

\section{Introduction}

Quality of life in city is an unmeasurable quantity, but citizens are able to feel it. Smart City is a concept, more than an architecture: technologies must improve well-being of citizens. Citizen well-being and life quality in general, depend on lot of factors, which are different in weight and in typology. Some of them like geographical location of the city, dimension, map, modernity of buildings and structures as well as the civic sense of the inhabitants are not easy to be controlled. On the contrary, others factors like public transportation, traffic and parking management, resource (energy, water, gas,) furniture and payment, social care of weakness, pollution, and waste disposal, fully belong to the sphere of responsibility of municipal authorities and companies that provide services on their behalf [1]-[2].

These fields today can be subject of great changes thanks to enabling IC technologies, which are being the main innovators. While digital networks provide the basic framework upon which smart cities make information flow, smart meters and all other peripheral devices [3]-[4] lie at the foundation of digital networks. Smart devices and digital networks together allow citizens to reach or to be reached from service utilities. Advanced Metering Infrastructures (AMIs) have been designed just for this, in the specific field of gas and water. Their Infrastructure typically includes: i) meters at the customer site; ii) the communication network between the customer and a service provider; iii) a management systems that makes the information available to the service provider.

As for AMIs numerous solutions have been experiencing until now, which adopted different physical channels and different communication protocols [5], [6]. Due to its high obstacle penetration capability and scarce power burden, the $169 \mathrm{MHz}$ wM-Bus is emerging as the most convincing physical channel for smart meters, even if the short range (350 $\mathrm{m})$ requires the installation of suitable gateways allowing smart meters to get to the Internet, via either fiber or GSMGPRS. Bandwidth is constrained in few kBytes, nevertheless it is wide enough for most of Smart City services. The low power radio module is compliant with battery powered device autonomy that should last at least five years. The wM-Bus, coupled with DLMS-COSEM [7]-[10] protocol, has been founding wide application as the best solution for communication and data structuring in gas field.

The problem is that one gateway would be able to manage the communication with some hundreds of smart RF devices, but the actual density of gas meters in urban area rarely get to this value in a radius of $300 \mathrm{~m}$. When the capability of gateways is not exploited properly, the costs to pay for each meter reading arises so much that gas utilities are discouraged from investing in AMIs. The solution could be that infrastructure cost be shared among more and more services that can be furnished through the same AMI.

While energy meters can trust the power line to be accessible and powered, water metering is quite far from this scenario. Even though some remote accessible solutions are market available, actually the most of installed meters are still mechanical. The major obstacle to wide spread is represented by cost and reliability of the newest measurement devices (i.e. ultrasound based meters) [11]-[12].

Other services are going to be experienced, like public illumination control but often through proprietary solutions. At first, the authors in [13] described a battery powered wireless device featured with a pollution sensor and that can connect to the $169 \mathrm{MHz}$ wM-Bus in AMI. In this paper the authors present the experience they are having in this field, where they are proposing some $169 \mathrm{MHz}$ wM-Bus based solutions for water metering, public car parking and elder teleassistance, to be added on the AMI they installed for gas meters. Some add-on devices were designed to extract information from mechanical meters, which take a digital 
picture of the meter display on demand, and transmit it to the gateway via $169 \mathrm{MHz}$ wM-bus radio module. Another battery powered device was designed to be held in car to pay parking in those public streets that are covered by a $169 \mathrm{MHz}$ wM-Bus gateway of the AMI. Finally also a solution to allow elders to send an alarm SMS to their relations just pushing a button, was made to be supported on the $169 \mathrm{MHz}$ net. The experimental test bed has been given by the city of Salerno (Italy), where more than 2500 devices are being experienced among gas, water, parking and elder assistance. After a description of the AMI and of the devices, results collected in the first year and half of experimentation will be briefly resumed.

\section{Advanced Metering Infrastructure in Smart City}

Smart metering and sharing of communication infrastructures are very topical concepts that have been the founding principles of the pilot project carried out in the municipality of Salerno, a city of about 160000 inhabitants in southern Italy. Smart metering is changing the relationship between the consumer and the supplier. Both home users and business users today demand that through IoT technologies they be provided with higher quality services without significant cost variation. Service providers, in turn, take advantage of smart technologies for optimized delivery and operational efficiency. From this point of view smart metering immediately appears as a win-win path, where you can have advantages for both counterparts.

The project pursued two objectives, to which utility companies tend with great interest:
- Meter-to-bill - the possibility to obtain - continuously useful information for billing from meter reading;

- Meter-to-grid - to be able to intelligently manage the network, by activating remote events that impact both the status and the configuration parameters of the smart devices themselves.

The AMI has been designed to make available the same effective means of communication to several services all characterized by different methods and amounts of data to be exchanged. On the infrastructure basis, an application has been designed to integrate both front and back office functions, with a portal to which the partners and the various actors access with different profiles. In addition to the remote reading of water and gas consumption, two other smart device types have been developed specifically for $169 \mathrm{MHz}$ wM-bus networks: one for the hourly payment of parking on public roads, and the other for remote assistance to the elderly. AMI was designed to collect measurements or events coming from smart devices and make them accessible to utility companies. Suitable gateways, called Concentrators, send data to and receive command from a Central Unit, which is featured with web interfaces suitably designed to allow any control strategy to be executed, and pricing data and commands to be sent to the various devices (see figure 1).

The AMI's communication architecture is characterized by hierarchical topology where leaf nodes (Smart Meters) are connected to master nodes (Concentrators). The leaf nodes are battery devices that perform medium or short range radio transmissions in wM-bus at $169 \mathrm{MHz}$ with a low power consumption, while master nodes add a GSM-GPRS modem (or a fiber cable connection to Internet) to get also long range communication capability. Every master node concentrates and transfers all data generated from a certain number of leaf

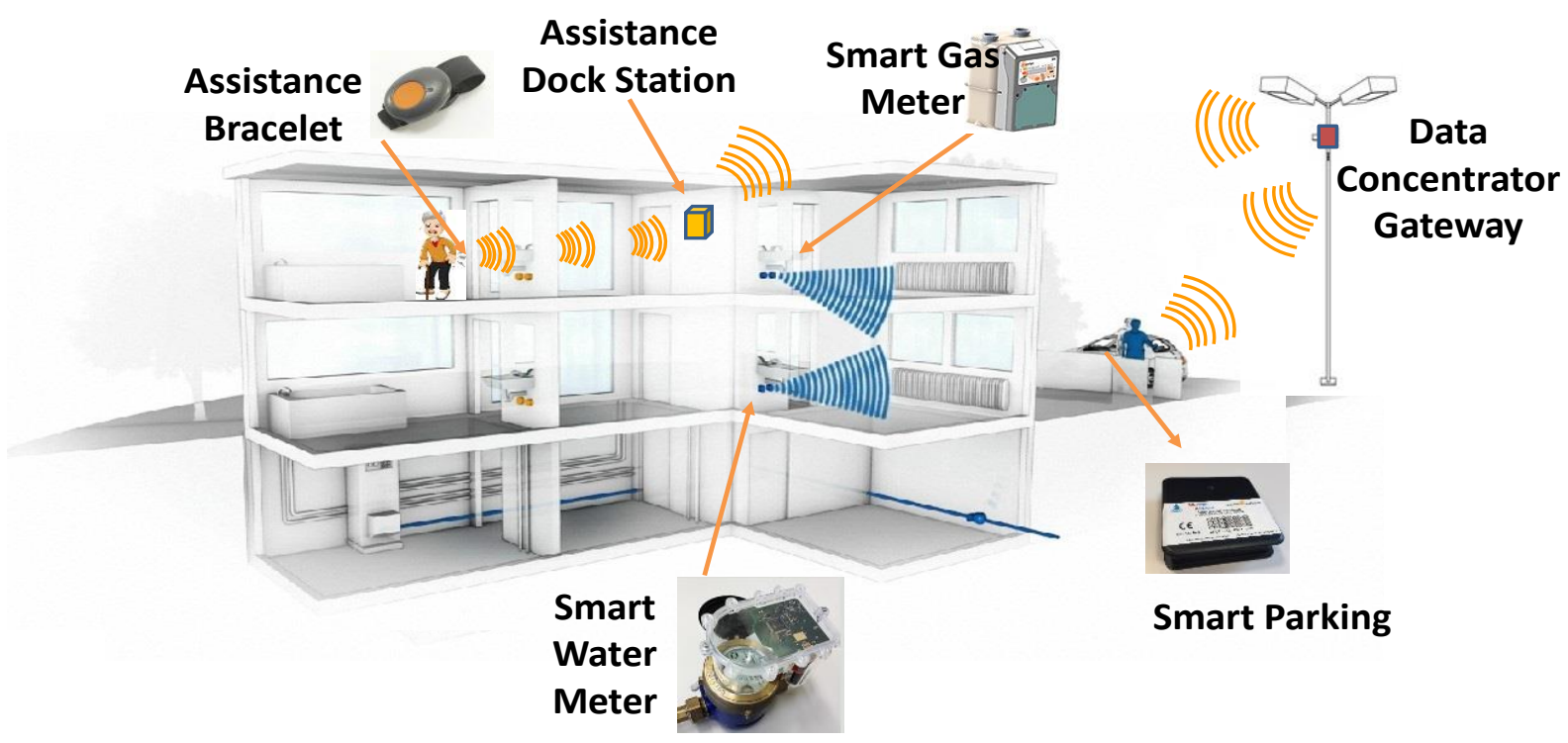

Figure 1 The Architecture of the AMI 
Table 1: List of smart devices and concentrators installed in the city of Salerno

\begin{tabular}{|c|c|c|c|c|c|c|c|c|}
\hline $\begin{array}{l}\text { City } \\
\text { Area }\end{array}$ & Address & Density & $\begin{array}{c}\mathrm{N}^{\circ} \text { of GAS } \\
\text { meters }\end{array}$ & $\begin{array}{c}\mathrm{N}^{\circ} \text { of Water } \\
\text { meters }\end{array}$ & $\begin{array}{c}\mathrm{N}^{\circ} \text { of Car } \\
\text { parking } \\
\text { sensors }\end{array}$ & $\begin{array}{l}\mathrm{N}^{\circ} \text { of Elderly } \\
\text { assistance }\end{array}$ & $\begin{array}{c}\text { Total } N^{\circ} \text { of } \\
\text { smart devices }\end{array}$ & $\begin{array}{c}\mathrm{N}^{\circ} \text { of } \\
\text { Concentrators }\end{array}$ \\
\hline 1 & $\begin{array}{l}\text { Via S. Nicola di } \\
\text { Giovi }\end{array}$ & Rural & 3 & 6 & - & - & 9 & 1 \\
\hline 2 & Via Tramontana & Rural & 10 & 14 & - & 1 & 25 & 1 \\
\hline 3 & Via Monticelli & Rural & 12 & 12 & - & 1 & 25 & 1 \\
\hline 4 & $\begin{array}{l}\text { Via Postiglione - } \\
\text { Ogliara }\end{array}$ & Suburban & 40 & 47 & - & 4 & 91 & 1 \\
\hline 5 & $\begin{array}{l}\text { Viale degli } \\
\text { Etruschi }\end{array}$ & Suburban & 40 & 56 & - & 4 & 100 & 1 \\
\hline 6 & Via S. Eustachio & Urban & 110 & 135 & - & 5 & 250 & 1 \\
\hline 7 & Via Passaro & Urban & 90 & 155 & - & 5 & 250 & 2 \\
\hline 8 & Via Seripando & $\begin{array}{c}\text { Densely } \\
\text { Urban }\end{array}$ & 95 & 90 & - & 15 & 200 & 1 \\
\hline 9 & Via Gaeta & $\begin{array}{l}\text { Densely } \\
\text { Urban }\end{array}$ & 125 & 160 & - & 15 & 300 & 1 \\
\hline 10 & P.zza M. Luciani & $\begin{array}{l}\text { Densely } \\
\text { Urban }\end{array}$ & 125 & 160 & 100 & 15 & 400 & 2 \\
\hline 11 & $\begin{array}{l}\text { Corso Giuseppe } \\
\text { Garibaldi }\end{array}$ & $\begin{array}{l}\text { Densely } \\
\text { Urban }\end{array}$ & 350 & 385 & 100 & 15 & 850 & 3 \\
\hline \multicolumn{3}{|r|}{ Total } & 1.000 & 1.220 & 200 & 80 & 2.500 & 15 \\
\hline
\end{tabular}

nodes. Usually, unlike leaf nodes, master nodes are either grid or solar cells powered. Both the technical feasibility and the economic convenience depend on the concentration ratio (number of smart devices for each concentrator). Since both these factors can be evaluated only experiencing urban areas characterized by different densities, at the first stage of the project, 11 city areas were identified, belonging to neighborhoods characterized by different population densities: rural, suburban, urban and densely urban. Smart devices and concentrators were spread in these 11 city areas, as listed in Table 1 and reported in details in figure 2.

With the objective of assessing the technical and economic feasibility of smart metering, the installation of the concentrators was carried out on available public buildings and sites and the choice of users to install the smart devices was determined by the coverage range of concentrators installed. All citizens were informed of experimentation through the media and those where the smart meters were installed were contacted directly by utility companies.

The pilot project final results will be analyzed in order to determine:

the effectiveness of the technology, in particular of the $169 \mathrm{MHz}$ RF physical channel;

the efficiency of smart metering respect to the traditional methods of reading in terms of economic sustainability and service quality perception;
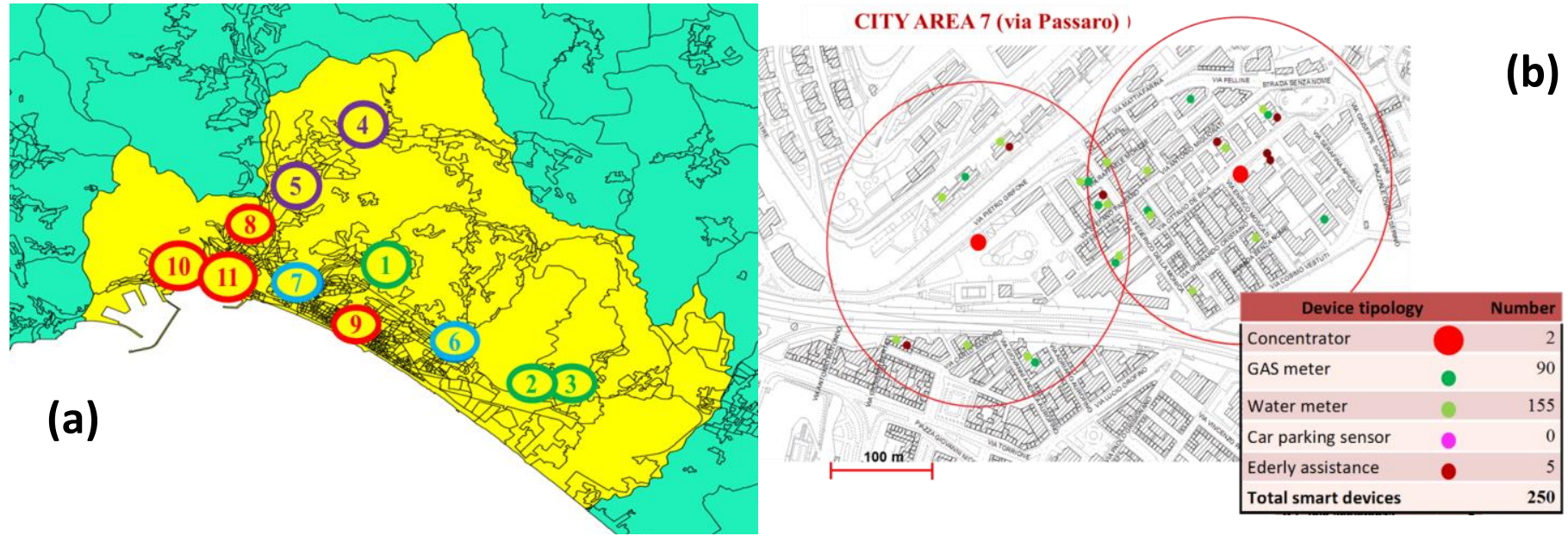

Figure 2 a) the 11 city areas in Salerno map; b) in the zoomed view of area $\mathbf{n . 7}$, the position of concentrators and meters 
the compatibility between metering and event based services (parking and elder assistance) when served by the same communication mean.

These conclusions are going to influence the future policy of public administration and utility companies and so the perspectives of the smart city trends in this ambit.

Furthermore the national authority for gas and energy is collecting the results of all the similar experiences carried out in the Italian country to update the regulations in the field.

\subsection{The $169 \mathrm{MHz}$ RF modules}

The infrastructure cost directly depends on the number of "concentrators" installed to cover the whole area of interest. As a consequence, the number of smart device connected to each concentrator (concentration ratio) is the main relative parameter to estimate the cost of services. The wider the coverage range of RF antenna, the higher the concentration ratio, the lower the costs. As for battery devices, transmission power and antenna gain must be compatible with battery capacity to assure long life of devices [13]. The wireless $M$ Bus (EN 13757-4:2013) is suggested by national authority because of its good tradeoff between coverage range and power requirements. The transmission mode, over $169 \mathrm{MHz}$ frequency band, allows good coverage range (hundreds of meters in urban area) due to the inherently lower path losses, while the reduced data rates permit higher sensitivity for the receiver, with a consequently reduction of the transmission power at the transmitter or a longer transmission range using the same transmission power [17]-[18]. The channel bandwidth $(7 \mathrm{kHz})$ is wide enough to allow both uplink and downlink (referred to the central unit). The transmission scheme is that leaf node starts the transmission to the master; after the transmission of the first packet, the master can send commands or requests, in a small reception time window. This means that battery devices can remain in sleep mode as long as they want, thus saving battery. If the leaf node receives a command/request in the reception window, it repeats the last message periodically with a determinate delay, until a new command/request is received from the master. The repetition stops for a termination message, or for timeout. Even though a physical channel like LoraWan would allow the same meter reachability and lightly longer coverage range at lower transmission power in uplink, it wouldn't give enough bandwidth for gas meters in downlink, for:

- configuration of meters;

- management of commercial parameters (consumption profiles, billing interval);

- setting of security parameters;

- meter firmware upgrade.

\subsection{Concentrator}

The Concentrator main tasks are collecting data from the
$169 \mathrm{MHz}$ smart devices, and sending them to the AMI Central Unit.

Concentrator architecture usually includes: i) a power PC board to processing information at application level (DCU functionalities); ii) a number of microcontroller-based radio modules equal to the number of simultaneous services (gas, electricity, water, etc...). At DLMS/COSEM protocol level, the Concentrator is both client (with respect to smart devices) and server (with respect to the central unit), and grants a transparent end-to-end communication.

The DLMS/COSEM communication profile is implemented by the concentrator also over TCP/IP (developed for GPRS transmission within a Public Network).

\subsection{The Central Unit}

Data coming from smart devices are indispensable to utility companies for billing and service quality analysis. The Central Unit must be addressable via the Internet by utilities that need to download data from smart devices and to upload commands.

The Central Unit consists of three software modules: i) a JAVA module that implements the DLMS-COSEM protocol to communicate with concentrators through the mobile (GPRS/UMTS) network; ii) a web application developed in PHP that allows users and utilities to send commands to the smart devices and access the stored data; iii) a MySQL relational database that records all data uploaded by the smart devices.

Also end customers may access their own data by adopting a user-friendly web interface, which makes the underlying communication protocols and objects transparent. About the security features, the Central Access System implements mechanisms for authentication to allow access to the concentrators and utilities.

The data is exchanged among head-end system and concentrators, whereas utilities are encrypted with AES-GCM 128 bit. It is ideal for protecting packetized data, because it has minimum latency and minimum operation overhead.

\section{Smart add-on for water meters}

The authors designed a smart device that has to be added on traditional analogue water meters to extract the reading from the photo of the front panel (see Figure 3).

The method allows the device to be released from any metrological specifications. Its enclosure has been designed to allow easy installation (without detachment) on a good number of meter models, and to let the water meter readable by customers. The smart add-on is made of a microcontroller (ARM®Cortex ${ }^{\mathrm{TM}}-\mathrm{M} 4$ 32-bit RISC core) based system, featured with the following devices: 


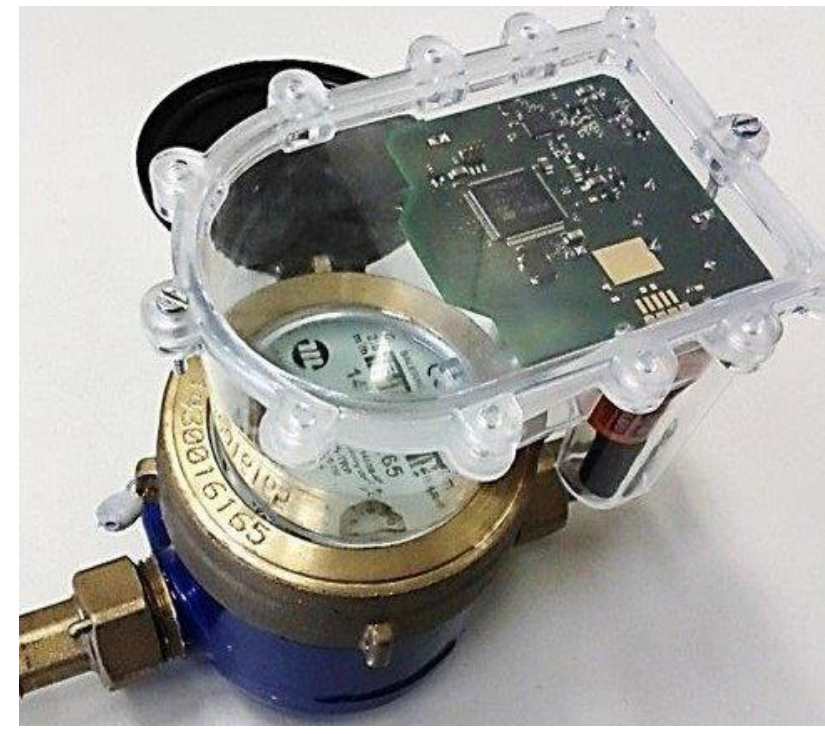

Figure 3 The smart add-on for water meters.

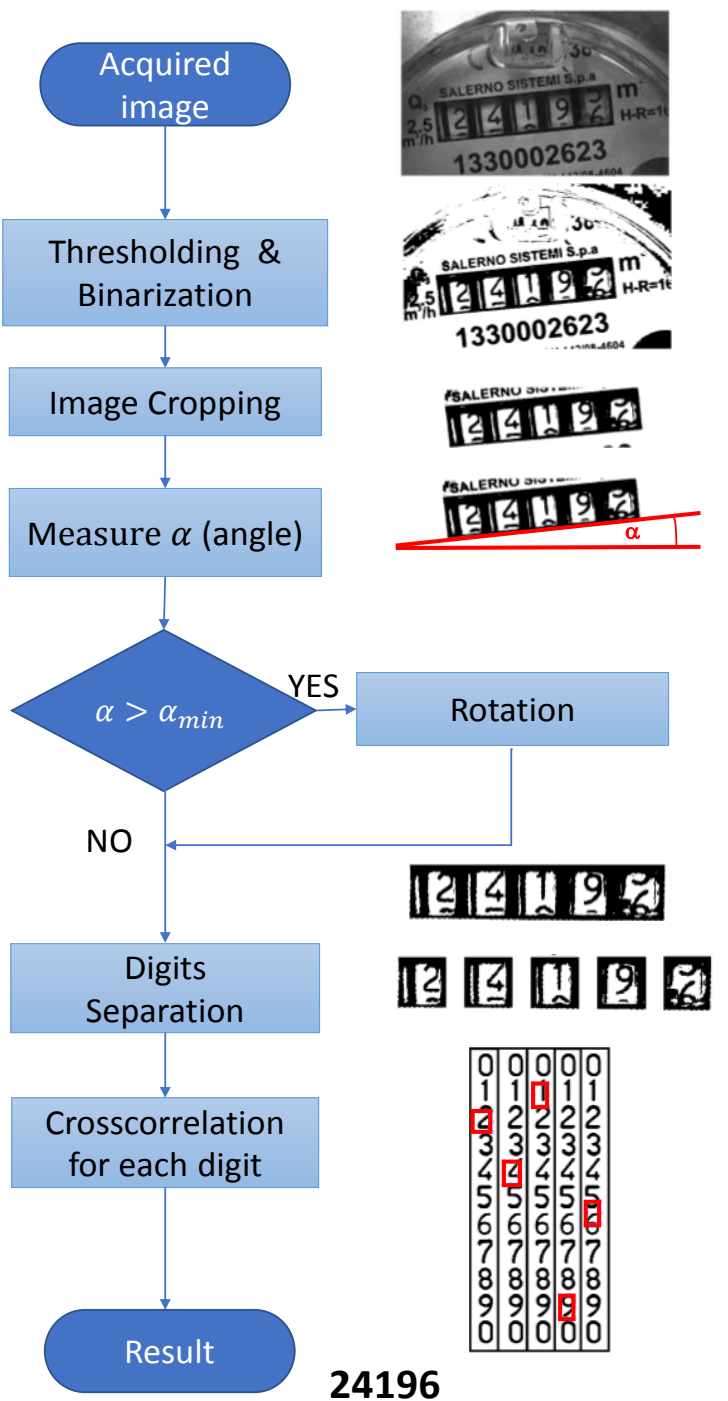

- $\quad$ Digital micro-camera with a field of view that includes consumption digits and serial number;

$169 \mathrm{MHz}$ wM-Bus RF module with antenna;

Battery power supply;

Containment enclosure made of transparent plastic material.

The DLMS-COSEM is implemented in the firmware thus making the device full compliant with the most widespread concentrators. Autonomous power supply (lithium battery with slow discharge), ensures a useful life of the device not less than 5 years. Radio-frequency channel transmission is not subject to government concession and the transmission distance is higher than $150 \mathrm{~m}$ in urban environment. On demand the photo can be requested by the concentrator. The meter will send the part of the binarized image that includes the five digits (see figure 4). It takes 11 data packets and digits can be either read by human operator, or extracted by an of line OCR software module, which has been integrated with the SAC web interface.

In its last release, the smart add-on firmware implements also an OCR feature that allows the five digits to be on-line extracted from the image and transformed in a numerical value of the consumption. After the bitmap image has been downloaded from the camera, it can be processed by a six steps procedure (Figure 4). The procedure main steps are: a) image digitalization through an adaptive threshold; b) localization and extraction of the decimal counter from the image; c) measurement and correction of the rotation angle due to installation inaccuracy; d) digit extraction and separation; e) cross-correlation of each digit with a suitable pattern which was set up to hold into account also the different lens distortion among the five digits; f) numerical evaluation of consumption.

The consumption value takes 1 packet to be uploaded to the concentrator, but also when only the consumption has been transmitted to the Central Unit, the photo can still be requested by the utility through a further command.

As for the performance of the OCR feature, it is available a statistic about only 130 readings, all made in the last two months by add-ons upgraded to the last release. It has to be highlighted that: i) the light conditions should be determined only by the on board white led because all smart water meters have been programmed to take pictures and transmit packets during the night, whilst daily times have been reserved to gas meters; ii) the relative position of the add-on respect to the meter display is forced during installation by a suitable hollow on the lower face of the add on, corresponding to a protrusion on the water meter display; iii) the camera focus is optimal in case of perfect installation.

Nevertheless in some cases (14\% of the total) these three conditions were not fully satisfied. The percentage of correct extraction was $90 \%$ and all the remaining $10 \%$ belongs to the $14 \%$ of installation errors.

Figure 4 On board OCR procedure 


\section{Elderly assistance Service}

The Multi-service communication infrastructure is used to support an elder tele-assistance service, with the aim to protect the wellbeing of users (mainly elderly and disabled people), allowing them a more serene stay in their living environment. The realized "Tele-assistance" service is able to generate an alarm for any type of emergency, active 24 hours a day, every day of the year.

The user is provided with:

- a push-button radio remote control (radio-frequency battery device in free loan) to generate the intervention request: for safety the user must wear or carry on the button;

- a dock-Station (the Sensor for Tele-Assistance) powered by an electrical source with dual radio communication, towards radio remote control and towards the nearest Concentrator, according to the $169 \mathrm{MHz}$ wM-Bus and DLMS / COSEM protocol.

The dock-station is then able to connect to the Multiservice

Concentrator to route the request for assistance to the Central Unit that collects all data coming from Concentrators. Finally, the Central Unit activates an Automatic Alert Report (through appropriate pre-recorded SMS message) in favor of the elder's trustworthy peoples (listed during registration to the service). The Dock-Station is programmed to send an acoustic luminous signal of absence of the service, both when the radio link with the Concentrator is interrupted, and when the Concentrator can not communicate via GSM-GPRS with the Central Unit. Other similar systems are available on the market, which send sms messages to registered numbers. All of them require either a wi-fi connection, or a land/cell phone line. The device proposed by the authors works within the coverage range of an installed Concentrator, without requiring direct access to the Internet or a telephone connection.

\section{Tele- parking service}

The Multi-service communication infrastructure is used also for managing the Public Parking slots (blue strips, gates) in order to improve both the perceived quality of the service (ease of access) and the efficiency of the provider. The TeleManagement service works thanks to a battery powered device that must be kept in the car by the user: a radio-remote control featured with a button that must be pushed when the car has been parked to communicate its identification code to the nearest Concentrator according to the wM-Bus and DLMS / COSEM protocol. The equipment is provided, on loan, to the user at the time of registration at the tele-parking service.

The starting of the parking time is activated manually, by the user by means of the push button: the remote terminal communicates at the Multiservice Concentrator which provides to route the request to start at the Central Unit. The request activates the system for charging the service provided. The end of the stay is automatically detected by the loosing of the connection between the remote terminal and the Multiservice Concentrator, when the car leaves. This condition indicates to the Central Units the end of service and allows the corresponding payment to be computed. In comparison with the parking platforms present on the market today, based on the adoption of SMS messages and/or smartphone applications, the proposed solution offers the advantage of greater simplicity and speed of access. No position, no expected duration, no information must be given, just a button push. The payment stops with max delay equal to the resolution of the Tele-parking (10 minutes) is determined automatically by the movement of the car with radio remote control still in ON state since the new spontaneous transmission that is of the addressed type will NOT receive acknowledgment from an eventual different concentrator visible from the radio-remote control during the movement.

The user must be sure only to stay in the coverage range of a Concentrator. The status of the connection is signaled by a

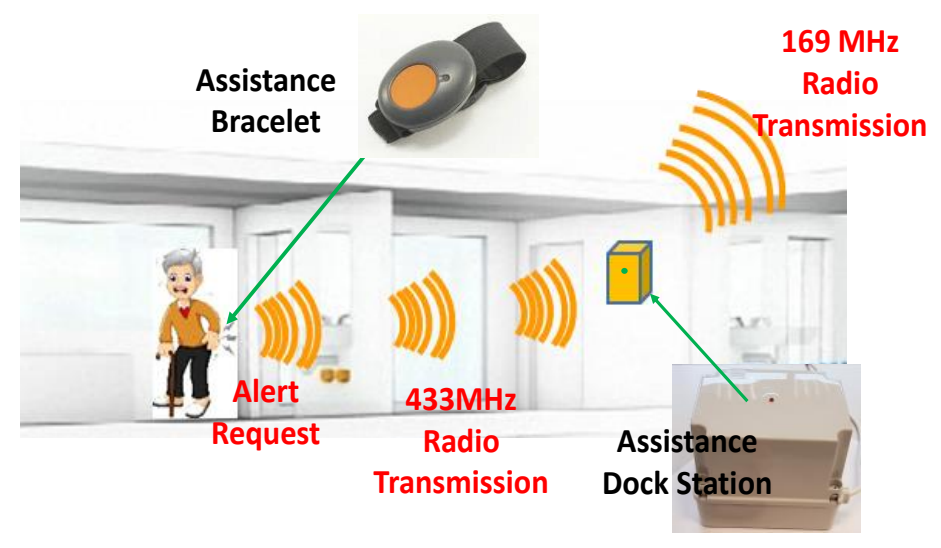

Figure 5. The smart devices for elder tele-assistance

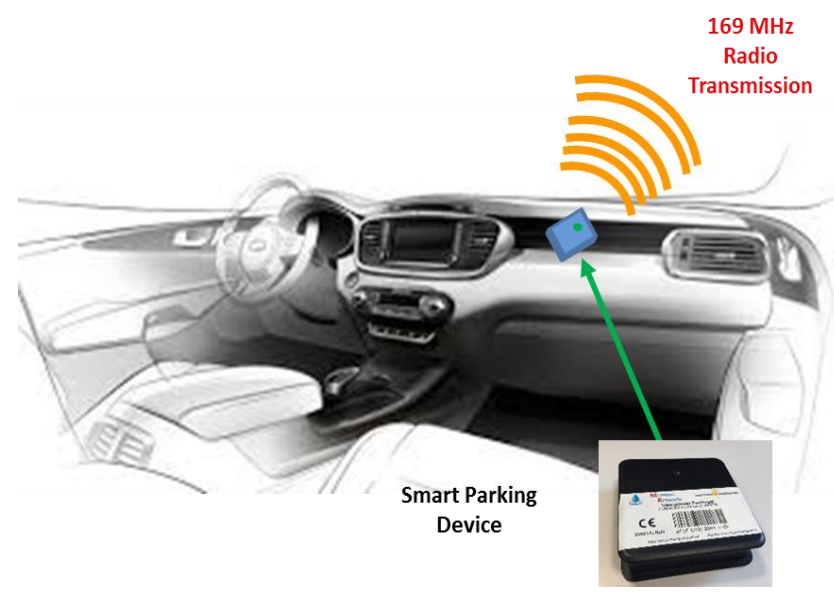

Figure 6 The Tele-parking device 

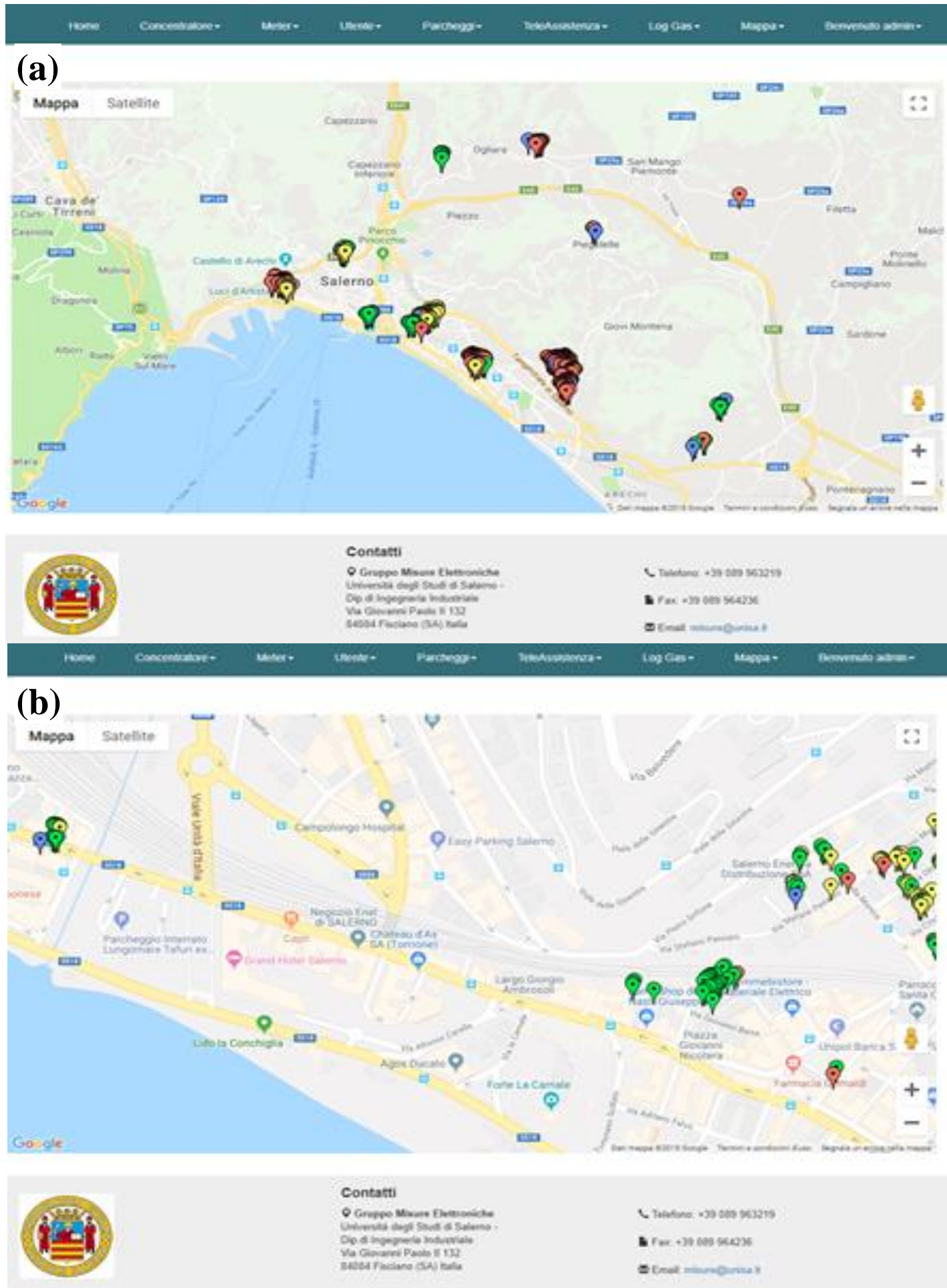

Figure 7 Two screen shots of the SAC web interface: a) all the map; b) zoomed part of the map.

suitable multi-color led and the average waiting for connection time is about 1 minute. The device must always be visible on the dashboard of the car and if the user don't push the button the multicolor remain turned of thus allowing controllers be alerted.

\section{Results}

The experimentation in the City of Salerno, started at the end of 2015, in November, and is still ongoing. The AMI has been installed between November 2015 and July 2016. It is made of 15 Concentrators, which were collocated in 11 city's 
Table 2 Summary of Application requirements

\begin{tabular}{|c|c|c|c|c|c|c|c|}
\hline \multirow[b]{2}{*}{ Application } & \multirow{2}{*}{$\begin{array}{l}\text { End- } \\
\text { points }\end{array}$} & \multicolumn{3}{|c|}{ Uplink } & \multicolumn{3}{|c|}{ Downlink } \\
\hline & & Periodicity & $\begin{array}{c}\text { Dataset } \\
\text { (bytes) }\end{array}$ & $\begin{array}{c}\text { Daily load } \\
\text { (bytes) }\end{array}$ & Periodicity & $\begin{array}{c}\text { Dataset } \\
\text { (bytes) }\end{array}$ & $\begin{array}{c}\text { Daily load } \\
\text { (bytes) }\end{array}$ \\
\hline Gas Metering & 1.000 & 3times/day & 150 & 450 & 1 time/month & 50 & - \\
\hline Water Metering & 1.200 & 3 time/day & 150 & 450 & 1 time/month & 50 & - \\
\hline Parking Management & 200 & 6 times/hour & 50 & 300 & 6 times/hour & 50 & 3 \\
\hline Health Alert & 80 & random & 50 & - & random & 50 & - \\
\hline Electricity Metering & 20 & 3times/day & 150 & 450 & 1 time/month & 50 & - \\
\hline
\end{tabular}

districts characterized by different densities of customers and different typology of buildings. Two screen shots of the SAC web interface are in figure 7. It is possible to note that the network manager profile allows the real time visualization of the status of meters through the different color they appear on the map. In particular, the green color means that meter transmitted last data within a week, whilst the yellow within a month and the red one more than a month. It's to be considered that water consumption is requested every two months, whilst gas meters transmit four times a day.

Experimental results have been collecting in the Central Unit Data Base and for sake of brevity were summarized in table 2 , in table 3 , and in table 4 . In table 2 the average data rate of each smart device is reported, both in uplink and in downlink (referred to the Central Unit). Uplink is periodic only for gas and water meters and concerns consumptions; whilst for the other services the data flow is unpredictable. Downlink is generally reserved to commands to be sent to

Table 3 Performance indices for Gas and Water Meter in terms of Reachability (R) and SAC (A).

\begin{tabular}{|c|c|c|}
\hline \multirow{2}{*}{ Index } & Gas & Water \\
\cline { 2 - 3 } & R/A & R/A \\
\hline Number of working points at the start & $1^{\prime} 040$ & $1^{\prime} 200$ \\
\hline Montly average reachability DAILY rate & $91 \%$ & $89 \%$ \\
\hline Montly average reachability WEAKLY rate & $97 \%$ & $95 \%$ \\
\hline Reachability rate in the MONTH & $99 \%$ & $97 \%$ \\
\hline
\end{tabular}

Table 4 Performance indices for Gas and Water Meter in terms of Reachability (R) and SAC Availability (A).

\begin{tabular}{|c|c|c|}
\hline \multirow{2}{*}{ Index } & Gas & Water \\
\cline { 2 - 3 } & R/A & R/A \\
\hline Number of working points at the start & $1^{\prime} 040$ & $1^{\prime} 200$ \\
\hline Montly average reachability DAILY rate & $91 \%$ & $87 \%$ \\
\hline Montly average reachability WEEK rate & $93 \%$ & $93 \%$ \\
\hline Reachability rate in the MONTH & $96 \%$ & $95 \%$ \\
\hline
\end{tabular}

devices for either commands of firmware upgrade.

In table 3 and table 4 , the report of reachability at both concentrator and Central Unit level of gas and water meters is showed at the early and the today stage of experimentation respectively.

The missing meters are still a few, after 24 months. The value let hope that battery capacity be enough to get to the expected goal of 5 years battery life for the most of devices. The behavior of parking and elder assistance devices has been more difficult to characterize until now, because their number is too low to produce significant statistics in so few months. However the customers questioned by phone by the staff of the utility company, said they were satisfied.

\section{Conclusions}

The AMI designed, implemented and experienced by the authors in the City of Salerno demonstrated the feasibility of the approach to Smart City context. All devices showed to be well designed to provide reliable services to customers. Battery devices exhibited enough autonomy to make the money investment convenient. All devices adopt DLMSCOSEM protocol, which leaves very few points of ambiguity in communication if its implementation is rigorous and complete. The occupation of the channel showed to be compatible with all the needs, also when all the services are fully working. Among these, the highest amount of transmitted data is due to a smart add-on for water meters. However, its newest release strongly reduces the number of packets to be transmitted to the concentrator thanks to an on board OCR feature, that demonstrated very good performance in case of right installation of the device. Elderly teleassistance ad tele parking devices look like being effective but will be useful when the RF network coverage will be extended to the whole city. The result of experimentation let imagine that in the near future these devices and infrastructure can be widespread. Battery device future developments will be focused on increasing autonomy and reducing dimension. As for the water smart meter, future efforts will be dedicated to reduce the sensitivity of the add-on device to the installation inaccuracy. 


\section{Acknownledgements}

The experimentation would not have been done without support and contribution of "Salerno Energia Distribuzione SpA" management and personnel.

\section{References}

[1] B. David, Y. Chuantao, Y. Zhou, T. Xu, B. Zhang, H. Jin, R. Chalon, "SMART-CITY: Problematics, techniques and case studies," Proceedings 8th International Conference on Computing Technology and Information Management (ICCM), 2012, Vol. 1, pp. 168 - 174.

[2] S. Lan, M. Qilong, J. Du, "Architecture of Wireless Sensor Networks for Environmental Monitoring," Proceedings International Workshop on Geoscience and Remote Sensing 2008, Vol. 1, pp. 579 -582 .

[3] Ferrigno, L., Morello, R., Paciello, V., Pietrosanto, A., "Remote metering in public networks", 2013, Metrology and Measurement Systems, vol. 20, n. 4, pp. 705-714.

[4] R. R. Mohassel, A. Fung, F. Mohammadi, K. Raahemifar, "A survey on Advanced Metering Infrastructure", Electrical Power and Energy Systems 63, pp. 473-484, 2014.

[5] Capriglione, D., Ferrigno, L., Attianese, A., Pietrosanto, A., Paciello, V., "Experimental analysis of wireless sensor network synchronization protocols under real operating conditions", 2014, Lecture Notes in Electrical Engineering, 162 LNEE, pp. 529-533

[6] A. Razavi, M. Jahed, "Node Positioning and Lifetime Optimization for Wireless Body Area Networks" IEEE Sensors Journal 2017, Vol. 17, no. 14, pp. 4647-4660 OpenMeter: Open Public Extended Network metering; Deliverables. http://www.openmeter.com

[7] Attianese, A., Del Giudice, A., Landi, M., Paciello, V., Pietrosanto, A., "Synchronization of DLMS/COSEM sensor nodes", 2013, 19th IMEKO TC4 Symposium - Measurements of Electrical Quantities 2013 and 17th International Workshop on ADC and DAC Modelling and Testing, pp. 234-239.

[8] IEC, "Electricity metering - Data exchange for meter reading, tariff and load control - Part 47: COSEM transport layers for IPv6 networks," IEC 62056-47, November 2006.

[9] Di Leo, G., Liguori, C., Paciello, V., Pietrosanto, A., Sommella, P., "Smart meters in smart cities: An application of DLMS-COSEM on $169 \mathrm{MHz}$ WM-Bus", 2016, Lecture Notes in Electrical Engineering, 362, pp.735-746

[10] Carratù M., Ferro M., Paciello V., Pietrosanto A., Sommella P., "Performance Analysis of wM-Bus Networks for Smart Metering", 2017, IEEE Sensors Journal, vol. 17, n. 23, pp. 7849-7856

[11] Capriglione, D., Ferrigno, L., Giudice, A.D., Graditi, G., Paciello, V., Pietrosanto, A., "Performance analysis of WM-Busbased synchronization protocols in Sensor Networks", 2014, 20th IMEKO TC4 Symposium on Measurements of Electrical Quantities, pp. 265,271

[12] G. Di Leo, C. Liguori, V. Paciello, A. Pietrosanto, P. Sommella, "Towards visual smart metering exploiting wM-Bus and DLMS/COSEM", Proceedings of 2015 IEEE International Conference on Computational Intelligence and Virtual Environments for Measurement Systems and Applications, CIVEMSA 2015, Shenzhen; China; 12-14 June 2015.

[13] Carratù, M., Ferro, M., Pietrosanto, A. and Sommella, P.: Adopting Smart Metering RF Networks for particulate matter distributed measurements. In: IMEKO TC19 Symposium: Metrology on Environmental Instrumentation and Measurements (EnvIMEKO 17), pp. 50-55. Aguascalientes, Mexico (2017).

[14] S. Squartini, L. Gabrielli, M. Mencarelli, M. Pizzichini, S. Spinsante, F. Piazza, "Wireless M-Bus Sensor Nodes in Smart Water Grids: the Energy Issue", 2013 Fourth International Conference on Intelligent Control and Information Processing (ICICIP)., Beijing, China, June 9-11, 2013, Vol. 8, N 7, pp. 614-619.

[15] Ferrigno, L., Paciello, V., Pietrosanto, A., "Performance characterization of a wireless instrumentation bus", 2010, IEEE Transactions on Instrumentation and Measurement, Vol. 59, n. 12, pp.3253-3261

[16] D. Capriglione, L. Ferrigno, E. D'Orazio, V. Paciello, A. Pietrosanto "Reliability analysis of RSSI for localization in small scale WSNs" 2012 IEEE International Instrumentation and Measurement Technology Conference Proceedings, 13-16 May 2012, Graz, Austria, pp. 935-940.

[17] Ferrigno, L., Pietrosanto, A., Paciello, V., "Low-cost visual sensor node for blue tooth-based measurement networks", 2006, IEEE Transactions on Instrumentation and Measurement, vol.55, n. 2, pp. 521-527.

[18] Di Leo, G., Liguori, C., Paciello, V., Pietrosanto, A., Sommella, P., "Towards visual smart metering exploiting wM-Bus and DLMS/COSEM", 2015, 2015 IEEE International Conference on Computational Intelligence and Virtual Environments for Measurement Systems and Applications, CIVEMSA 2015. 